\title{
On a theorem of Bombieri-Vinogradov type for prime numbers from a thin set
}

\author{
by
}

D. I. Tolev (Plovdiv)

In 1940 I. M. Vinogradov considered the set

$$
S_{\lambda}=\left\{p \text { prime } \mid\{\sqrt{p}\}<p^{-\lambda}\right\},
$$

where $\lambda>0$ is a fixed number and $\{t\}$ denotes the fractional part of $t$. Vinogradov proved ([17], Chapter 4 ) that if $0<\lambda<1 / 10$ then

$$
\sum_{p \leq x, p \in S_{\lambda}} 1 \sim \frac{x^{1-\lambda}}{(1-\lambda) \log x} \quad \text { as } x \rightarrow \infty .
$$

A different approach to this problem was developed by Linnik [11] in 1945. In 1979 Kaufman [10] used the method of Linnik and proved the asymptotic formula (1) for $\lambda<0.1631 \ldots$ He also proved that if the Riemann Hypothesis is assumed then (1) holds for $\lambda<1 / 4$.

In 1983 Balog [1] and Harman [8] used Vaughan's identity and mean value estimates for Dirichlet polynomials and independently proved without assuming the Riemann Hypothesis that the formula (1) is true for $\lambda<$ 1/4. Later Balog [2] generalized his result to prime numbers in arithmetic progressions. We should also mention the works of Schoissengeier [14], [15], Gritsenko [7] and Rivat [13].

In the present paper we use the method of Balog and Harman and we prove a theorem of Bombieri-Vinogradov type for prime numbers from the set $S_{\lambda}$.

Let $\lambda, \theta$ be real numbers such that

$$
0<\lambda<1 / 4, \quad 0<\theta<1 / 4-\lambda .
$$

Let $x$ be a sufficiently large real number, $\mathcal{L}=\log x ; y, u, v, t, \alpha, \nu, \tau, V, Y$, $K, M, N, D$ real numbers; $a, k, l, m, n$ integers; $A$ an arbitrarily large positive

1991 Mathematics Subject Classification: Primary 11N13.

Research done with the financial support of Bulgarian Ministry of Education and Science, grant MM-430. 
number; $\varepsilon$ an arbitrarily small positive number. In formulas which do not involve $\varepsilon$ the constants in $O$-terms and $\ll$-symbols are absolute or depend only on $A, \lambda, \theta$. In formulas which involve $\varepsilon$ the constants also depend on $\varepsilon$. As usual, $[t]$ denotes the integer part of $t, e(t)=e^{2 \pi i t} ; \mu(n), \Lambda(n), \varphi(n), \tau(n)$ denote Möbius' function, von Mangoldt's function, Euler's function and the number of positive divisors of $n$, respectively.

$\sum_{\chi \bmod k}$ denotes the sum over all characters $(\bmod k)$, and $\sum_{\chi \bmod k}^{*}$ the sum over all primitive characters $(\bmod k)$; finally,

$$
\psi_{\lambda}(y ; k, a)=\sum_{\substack{n \leq y \\ n \equiv a(\bmod k) \\\{\sqrt{n}\}<n^{-\lambda}}} \Lambda(n) .
$$

We prove the following theorem:

TheOREM. If $\lambda$ and $\theta$ satisfy (2) and $A>0$ is arbitrarily large then

$$
E=\sum_{k \leq x^{\theta}} \max _{y \leq x} \max _{(a, k)=1}\left|\psi_{\lambda}(y ; k, a)-\frac{y^{1-\lambda}}{\varphi(k)(1-\lambda)}\right| \ll x^{1-\lambda} \mathcal{L}^{-A} .
$$

Proof. We may suppose that $A>10$. Let $B=10 A$. If $k \leq x^{\theta}$ then using only a simple counting argument we find

$$
\psi_{\lambda}\left(x \mathcal{L}^{-B} ; k, a\right) \ll \mathcal{L} \sum_{\substack{n \leq x \mathcal{L}^{-B} \\ n \equiv a(\bmod k) \\\{\sqrt{n}\}<n^{-\lambda}}} 1 \ll k^{-1} x^{1-\lambda} \mathcal{L}^{2-B / 2} .
$$

Note that to prove the last estimate the upper bound for $\theta$ need not be so tight as in (2). The same happens in other places as well. We use the strong restriction $\theta<1 / 4-\lambda$ only at the end of the proof to obtain (38) and (39) from (36) and (37).

From (3) we get

$$
E \ll E_{1}+x^{1-\lambda} \mathcal{L}^{-A}
$$

where

$$
E_{1}=\sum_{k \leq x^{\theta}} \max _{x \mathcal{L}^{-B} \leq y \leq x} \max _{(a, k)=1}\left|\psi_{\lambda}(y ; k, a)-\frac{y^{1-\lambda}}{\varphi(k)(1-\lambda)}\right| .
$$

Define $u_{v}=v\left(1-(\log v)^{-B}\right)$, and

$$
S_{\lambda}^{*}(v ; k, a)=\sum_{\substack{u_{v}<n \leq v \\
n \equiv a(\bmod k) \\
\{\sqrt{n}\}<n^{-\lambda}}} \Lambda(n), \quad S_{\lambda}(v ; k, a)=\sum_{\begin{array}{c}
u_{v}<n \leq v \\
n \equiv a(\bmod k) \\
\{\sqrt{n}\}<\sqrt{n} v^{-1 / 2-\lambda}
\end{array}} \Lambda(n),
$$




$$
P_{\lambda}^{*}(v ; k, a)=\sum_{\substack{u_{v}<n \leq v \\ n \equiv a(\bmod k)}} \Lambda(n) n^{-\lambda}, \quad P_{\lambda}(v ; k, a)=v^{-1 / 2-\lambda} \sum_{\substack{u_{v}<n \leq v \\ n \equiv a(\bmod k)}} \Lambda(n) n^{1 / 2} .
$$

It is not difficult to see that if $x \mathcal{L}^{-2 B} \leq v \leq x$ and $k \leq x^{\theta}$ then

$$
\begin{aligned}
& S_{\lambda}^{*}(v ; k, a)-S_{\lambda}(v ; k, a) \ll k^{-1} x^{1-\lambda} \mathcal{L}^{1-2 B}, \\
& P_{\lambda}^{*}(v ; k, a)-P_{\lambda}(v ; k, a) \ll k^{-1} x^{1-\lambda} \mathcal{L}^{1-2 B} .
\end{aligned}
$$

Let us prove, for example, the first of the inequalities above. We have

$$
S_{\lambda}^{*}(v ; k, a)-S_{\lambda}(v ; k, a) \ll \mathcal{L} \sum_{\begin{array}{c}
u_{v}<n \leq v \\
n \equiv a(\bmod k) \\
\sqrt{n} v^{-1 / 2-\lambda} \leq\{\sqrt{n}\}<\sqrt{n} u_{v}^{-1 / 2-\lambda}
\end{array}} 1 .
$$

If $l^{2} \leq n<(l+1)^{2}$ and $\sqrt{n} v^{-1 / 2-\lambda} \leq\{\sqrt{n}\}<\sqrt{n} u_{v}^{-1 / 2-\lambda}$ then we have

$$
l^{2}\left(1-v^{-1 / 2-\lambda}\right)^{-2} \leq n<l^{2}\left(1-u_{v}^{-1 / 2-\lambda}\right)^{-2} .
$$

We use the definition of $u_{v}$ and the restriction imposed on $k$ and after some calculations we find that the expression being estimated is

$$
\begin{aligned}
& \ll \mathcal{L} \sum_{\sqrt{u_{v}}-1<l \leq \sqrt{v}}\left(1+k^{-1} l^{2}\left(\left(1-u_{v}^{-1 / 2-\lambda}\right)^{-2}-\left(1-v^{-1 / 2-\lambda}\right)^{-2}\right)\right) \\
& \ll k^{-1} x^{1-\lambda} \mathcal{L}^{1-2 B} .
\end{aligned}
$$

For each $y \in\left[x \mathcal{L}^{-B}, x\right]$ we define the sequence $y_{i}, 0 \leq i \leq i_{0}$, in the following way:

$$
y_{0}=y, \quad y_{i+1}=y_{i}\left(1-\left(\log y_{i}\right)^{-B}\right), \quad y_{i_{0}+1}<y(\log y)^{-B} \leq y_{i_{0}} .
$$

Clearly

$$
i_{0} \ll \mathcal{L}^{B+1} .
$$

If $y \in\left[x \mathcal{L}^{-B}, x\right]$ then using (3), (5)-(7) we get

$$
\begin{aligned}
\psi_{\lambda}(y ; k, a)- & \sum_{\substack{n \leq y \\
n \equiv a(\bmod k)}} \Lambda(n) n^{-\lambda} \\
& \ll \sum_{0 \leq i \leq i_{0}}\left(\left|S_{\lambda}^{*}\left(y_{i} ; k, a\right)-S_{\lambda}\left(y_{i} ; k, a\right)\right|+\left|S_{\lambda}\left(y_{i} ; k, a\right)-P_{\lambda}\left(y_{i} ; k, a\right)\right|\right. \\
& \left.\quad+\left|P_{\lambda}\left(y_{i} ; k, a\right)-P_{\lambda}^{*}\left(y_{i} ; k, a\right)\right|\right)+k^{-1} x^{1-\lambda} \mathcal{L}^{2-B / 2} \\
& \ll \sum_{0 \leq i \leq i_{0}}\left|S_{\lambda}\left(y_{i} ; k, a\right)-P_{\lambda}\left(y_{i} ; k, a\right)\right|+k^{-1} x^{1-\lambda} \mathcal{L}^{2-B / 2} .
\end{aligned}
$$


The last inequality and (7) imply

$$
E_{1} \ll E_{2}+\mathcal{L}^{B+1} E_{3}+x^{1-\lambda} \mathcal{L}^{-A},
$$

where

$$
\begin{aligned}
& E_{2}=\sum_{k \leq x^{\theta}} \max _{x \mathcal{L}^{-B} \leq y \leq x} \max _{(a, k)=1}\left|\sum_{\substack{n \leq y \\
n \equiv a(\bmod k)}} \Lambda(n) n^{-\lambda}-\frac{y^{1-\lambda}}{\varphi(k)(1-\lambda)}\right|, \\
& E_{3}=\sum_{k \leq x^{\theta}} \max _{\mathcal{L}^{-2 B} \leq v \leq x} \max _{(a, k)=1}\left|S_{\lambda}(v ; k, a)-P_{\lambda}(v ; k, a)\right| .
\end{aligned}
$$

We use the Bombieri-Vinogradov theorem [4] to obtain

$$
E_{2} \ll x^{1-\lambda} \mathcal{L}^{-A} \text {. }
$$

Obviously

$$
E_{3} \ll \mathcal{L} \max _{x \mathcal{L}^{-2 B} \leq V \leq x} E_{4}
$$

where

$$
E_{4}=E_{4}(V)=\sum_{k \leq x^{\theta}} \max _{V / 2 \leq v \leq V} \max _{(a, k)=1}\left|S_{\lambda}(v ; k, a)-P_{\lambda}(v ; k, a)\right| .
$$

Hence, using (4) and (8)-(10) we have

$$
E \ll \mathcal{L}^{B+2} \max _{x \mathcal{L}^{-2 B} \leq V \leq x} E_{4}+x^{1-\lambda} \mathcal{L}^{-A} .
$$

Suppose that

$$
\begin{aligned}
x \mathcal{L}^{-2 B} & \leq V \leq x, \quad V / 2 \leq v \leq V, \quad T=V^{1 / 2+\lambda} \mathcal{L}^{2 B}, \\
T_{0} & =V^{1 / 2} / 10, \quad k \leq x^{\theta}, \quad(a, k)=1 .
\end{aligned}
$$

Let $\chi$ be a character $(\bmod k)$. We define

$$
\begin{aligned}
& F(s)=\sum_{\substack{u_{v}<n \leq v \\
n \equiv a(\bmod k)}} \Lambda(n) n^{s}, \quad F_{\chi}(s)=\sum_{u_{v}<n \leq v} \chi(n) \Lambda(n) n^{s}, \\
& L(s)=\sum_{V^{1 / 2} / 10<l \leq 10 V^{1 / 2}} l^{-s}, \quad H(s)=\frac{1}{s}\left(1-\left(1-v^{-1 / 2-\lambda}\right)^{s}\right), \\
& I=\frac{1}{2 \pi i} \int_{1 / 2-i T}^{1 / 2+i T} F(s / 2) L(s) H(s) d s, \quad I_{0}=\frac{1}{2 \pi i} \int_{1 / 2-i T_{0}}^{1 / 2+i T_{0}} F(s / 2) L(s) H(s) d s .
\end{aligned}
$$

We use Perron's formula $([6], \S 17)$ to get

$$
S_{\lambda}(v ; k, a)=\sum_{\substack{u_{v}<n \leq v \\ n \equiv a(\bmod k)}} \Lambda(n)\left([\sqrt{n}]-\left[\sqrt{n}\left(1-v^{-1 / 2-\lambda}\right)\right]\right)
$$




$$
\begin{aligned}
= & \sum_{\substack{u_{v}<n \leq v \\
n \equiv a(\bmod k)}} \Lambda(n) \\
& \times \sum_{V^{1 / 2} / 10<l \leq 10 V^{1 / 2}}\left(\frac{1}{2 \pi i} \int_{1 / 2-i T}^{1 / 2+i T} n^{s / 2} l^{-s} H(s) d s\right. \\
& +O\left(\min \left(1, T^{-1}\left|\log \frac{\sqrt{n}}{l}\right|^{-1}\right)\right. \\
& \left.\left.+\min \left(1, T^{-1}\left|\log \frac{\sqrt{n}}{l}\left(1-v^{-1 / 2-\lambda}\right)\right|^{-1}\right)\right)\right) \\
= & I+O\left(\mathcal{L}\left(\Delta_{1}+\Delta_{2}\right)\right),
\end{aligned}
$$

where

$$
\begin{aligned}
\Delta_{1} & =\sum_{\substack{V / 4 \leq n \leq V \\
n \equiv a(\bmod k)}} \sum_{V^{1 / 2} / 10<l \leq 10 V^{1 / 2}} \min \left(1, T^{-1}\left|\log \frac{\sqrt{n}}{l}\right|^{-1}\right), \\
\Delta_{2} & =\sum_{\substack{V / 4 \leq n \leq V \\
n \equiv a(\bmod k)}} \sum_{V^{1 / 2} / 10<l \leq 10 V^{1 / 2}} \min \left(1, T^{-1}\left|\log \frac{\sqrt{n}}{l}\left(1-v^{-1 / 2-\lambda}\right)\right|^{-1}\right) .
\end{aligned}
$$

We use (12) and after some standard calculations we obtain

$$
\Delta_{1}, \Delta_{2} \ll k^{-1} x^{1-\lambda} \mathcal{L}^{2-2 B}
$$

If $s=1 / 2+i t,|t| \leq T_{0}$ then we may approximate the exponential sum $L(s)$ by an integral ([9], Chapter III, $\S 1$, Corollary 1$)$ to get

$$
L(s)=\frac{\left(10 V^{1 / 2}\right)^{1-s}-\left(V^{1 / 2} / 10\right)^{1-s}}{1-s}+O\left(V^{-1 / 4}\right)=O\left(\frac{V^{1 / 4}}{1+|t|}\right) .
$$

We also have

$$
H(s) \ll V^{-1 / 2-\lambda}, \quad H(s)=v^{-1 / 2-\lambda}+O\left(|s-1| V^{-1-2 \lambda}\right) .
$$

Hence

$$
\begin{aligned}
I_{0}= & \frac{v^{-1 / 2-\lambda}}{2 \pi i} \int_{1 / 2-i T_{0}}^{1 / 2+i T_{0}} F(s / 2) \frac{\left(10 V^{1 / 2}\right)^{1-s}-\left(V^{1 / 2} / 10\right)^{1-s}}{1-s} d s \\
& +O\left(V^{-3 / 4-\lambda} \int_{-T_{0}}^{T_{0}}\left|F\left(\frac{1}{4}+\frac{1}{2} i t\right)\right| d t\right) .
\end{aligned}
$$

Using the orthogonality of characters $(\bmod k)$, Cauchy's inequality and Theorem 6.1 of [12] we get 


$$
\begin{aligned}
\int_{-T_{0}}^{T_{0}}\left|F\left(\frac{1}{4}+\frac{1}{2} i t\right)\right| d t & \ll \frac{1}{\varphi(k)} \sum_{\chi \bmod k} \int_{-T_{0}}^{T_{0}}\left|F_{\chi}\left(\frac{1}{4}+\frac{1}{2} i t\right)\right| d t \\
& \ll \frac{1}{\varphi(k)} \sum_{\chi \bmod k} T_{0}^{1 / 2}\left(\int_{-T_{0}}^{T_{0}}\left|F_{\chi}\left(\frac{1}{4}+\frac{1}{2} i t\right)\right|^{2} d t\right)^{1 / 2} \\
& \ll x^{3 / 2} \mathcal{L} .
\end{aligned}
$$

We substitute the last estimate in (16) and apply Perron's formula again. We get

$$
I_{0}=P_{\lambda}(v ; k, a)+O\left(k^{-1} x^{1-\lambda} \mathcal{L}^{-2 B}\right) .
$$

From $(13)-(17)$ and from the orthogonality of the characters $(\bmod k)$ we obtain

$$
\begin{aligned}
& S_{\lambda}(v ; k, a)-P_{\lambda}(v ; k, a) \\
& \ll k^{-1} x^{1-\lambda} \mathcal{L}^{3-2 B}+\frac{V^{-1 / 2-\lambda}}{\varphi(k)} \sum_{\chi \bmod k} \int_{T_{0}}^{T}\left|F_{\chi}\left(\frac{1}{4}+\frac{1}{2} i t\right)\right| \cdot\left|L\left(\frac{1}{2}+i t\right)\right| d t .
\end{aligned}
$$

The last estimate and (11) imply

$$
E \ll \mathcal{L}^{B+3} \max _{x \mathcal{L}^{-2 B} \leq V \leq x}\left(V^{-1 / 2-\lambda} E_{5}\right)+x^{1-\lambda} \mathcal{L}^{-A},
$$

where

$$
E_{5}=E_{5}(V)=\sum_{k \leq x^{\theta}} \frac{1}{k} \sum_{\chi \bmod k} \max _{V / 2 \leq v \leq V} \int_{T_{0}}^{T}\left|F_{\chi}\left(\frac{1}{4}+\frac{1}{2} i t\right)\right| \cdot\left|L\left(\frac{1}{2}+i t\right)\right| d t .
$$

We approximate the exponential sum $L$ in the last expression by a shorter one ([9], Chapter III, $\S 1$, Theorem 1) and we obtain

$$
\left|L\left(\frac{1}{2}+i t\right)\right| \ll 1+\left|\sum_{t V^{-1 / 2} /(20 \pi)<l \leq 5 t V^{-1 / 2} / \pi} l^{-1 / 2-i t}\right|=1+\left|L_{1}(t)\right|,
$$

say. Hence we have

$$
\begin{aligned}
E_{5} & \ll \sum_{k \leq x^{\theta}} \frac{1}{k} \sum_{\chi \bmod k} \max _{V / 2 \leq v \leq V} \int_{T_{0}}^{T}\left|F_{\chi}\left(\frac{1}{4}+\frac{1}{2} i t\right)\right|\left(1+\left|L_{1}(t)\right|\right) d t \\
& \ll \mathcal{L} \max _{x^{1 / 2} \mathcal{L}^{-1-B} \leq Y \leq x^{1 / 2+\lambda} \mathcal{L}^{2 B}} E_{6},
\end{aligned}
$$

where

$$
E_{6}=E_{6}(V, Y)=\sum_{k \leq x^{\theta}} \frac{1}{k} \sum_{\chi \bmod k} \max _{V / 2 \leq v \leq V} \int_{Y / 2}^{Y}\left|F_{\chi}\left(\frac{1}{4}+\frac{1}{2} i t\right)\right|\left(1+\left|L_{1}(t)\right|\right) d t .
$$


The interval of summation in $L_{1}(t)$ depends on $t$. To get rid of this dependence we apply, for example, Lemma 2.2 of [5] to get

$$
\left|L_{1}(t)\right| \ll \int_{-\infty}^{\infty} K(\alpha)\left|L_{2}(t, \alpha)\right| d \alpha
$$

where

$$
L_{2}(t, \alpha)=\sum_{Y V^{-1 / 2} / 200<l \leq 2 Y V^{-1 / 2}} e(\alpha l) l^{-1 / 2-i t}
$$

and where the kernel $K(\alpha)$ depends only on $\alpha, Y, V$ and satisfies the inequalities

$$
K(\alpha) \geq 0, \quad 1 \ll \int_{-\infty}^{\infty} K(\alpha) d \alpha \ll \mathcal{L}
$$

Therefore

$$
E_{6} \ll \mathcal{L} \max _{0 \leq \alpha \leq 1} E_{7}
$$

where

$$
\begin{aligned}
E_{7} & =E_{7}(V, Y, \alpha) \\
& =\sum_{k \leq x^{\theta}} \frac{1}{k} \sum_{\chi \bmod k} \max _{V / 2 \leq v \leq V} \int_{Y / 2}^{Y}\left|F_{\chi}\left(\frac{1}{4}+\frac{1}{2} i t\right)\right|\left(1+\left|L_{2}(t, \alpha)\right|\right) d t .
\end{aligned}
$$

We use the properties of primitive characters and the inequality

$$
\int_{Y / 2}^{Y}\left|L_{2}(t, \alpha)\right| d t \ll Y,
$$

which is a consequence of Cauchy's inequality and Theorem 6.1 of [12]. After some calculations we get

$$
E_{7} \ll \mathcal{L}\left(E_{8}+E_{9}\right)+x
$$

where

$$
\begin{aligned}
E_{8} & =E_{8}(V, Y, \alpha)=\max _{V / 2 \leq v \leq V} \int_{Y / 2}^{Y}\left|\sum_{u_{v}<n \leq v} \Lambda(n) n^{1 / 4+i t / 2}\right|\left(1+\left|L_{2}(t, \alpha)\right|\right) d t, \\
E_{9} & =E_{9}(V, Y, \alpha) \\
& =\sum_{k \leq x^{\theta}} \frac{1}{k} \sum_{\chi \bmod k}^{*} \max _{V / 2 \leq v \leq V} \int_{Y / 2}^{Y}\left|F_{\chi}\left(\frac{1}{4}+\frac{1}{2} i t\right)\right|\left(1+\left|L_{2}(t, \alpha)\right|\right) d t .
\end{aligned}
$$

It remains to prove that if $V$ and $Y$ satisfy the conditions imposed in (18), (19) then we have

$$
E_{8}, E_{9} \ll x^{3 / 2-\nu}
$$


for some $\nu>0$. The proof of the theorem follows from (18)-(20), (22), (23).

Let us consider $E_{9}$. The estimation of $E_{8}$ is similar and, in fact, it was done in [1]. Clearly

$$
E_{9} \ll \mathcal{L} \max _{K \leq x^{\theta}}\left(K^{-1} E_{10}\right),
$$

where

$$
\begin{aligned}
E_{10} & =E_{10}(V, Y, \alpha, K) \\
& =\sum_{k \leq K} \sum_{\chi \bmod k}^{*} \max _{V / 2 \leq v \leq V} \int_{Y / 2}^{Y}\left|F_{\chi}\left(\frac{1}{4}+\frac{1}{2} i t\right)\right|\left(1+\left|L_{2}(t, \alpha)\right|\right) d t .
\end{aligned}
$$

Let

$$
D=x^{\lambda+(1-4 \lambda) / 400} .
$$

We apply Vaughan's identity [16] to get

$$
F_{\chi}\left(\frac{1}{4}+\frac{1}{2} i t\right)=F_{1}-F_{2}-F_{3}-F_{4},
$$

where

$$
\begin{aligned}
& F_{1}=\sum_{m \leq D} \sum_{u_{v} / m<n \leq v / m} \mu(m)(\log n) \chi(m n)(m n)^{1 / 4+i t / 2}, \\
& F_{2}=\sum_{m \leq D} \sum_{u_{v} / m<n \leq v / m} c(m) \chi(m n)(m n)^{1 / 4+i t / 2}, \\
& F_{3}=\sum_{D<m \leq D^{2}} \sum_{u_{v} / m<n \leq v / m} c(m) \chi(m n)(m n)^{1 / 4+i t / 2}, \\
& F_{4}=\sum_{u_{v}<m n \leq v, m, n>D} a(m) \Lambda(n) \chi(m n)(m n)^{1 / 4+i t / 2}, \\
&|c(m)| \leq \log m, \quad|a(m)| \leq \tau(m) .
\end{aligned}
$$

We have

$$
E_{10} \ll E_{10}^{(1)}+E_{10}^{(2)}+E_{10}^{(3)}+E_{10}^{(4)},
$$

where $E_{10}^{(i)}$ denotes the contribution to $E_{10}$ arising from $F_{i}$.

Let us consider $E_{10}^{(1)}$. We have

$$
F_{1}=\sum_{m \leq D} \mu(m) \chi(m) m^{1 / 4+i t / 2} W_{m},
$$


where

$$
\begin{aligned}
W_{m} & =\sum_{u_{v} / m<n \leq v / m}(\log n) \chi(n) n^{1 / 4+i t / 2} \\
& =\sum_{1 \leq l \leq k} \chi(l) \sum_{\substack{u_{v} / m<n \leq v / m \\
n \equiv l(\bmod k)}}(\log n) n^{1 / 4+i t / 2}=\sum_{1 \leq l \leq k} \chi(l) \Gamma_{l},
\end{aligned}
$$

say. We use (2) and (25) to conclude that the exponential sum $\Gamma_{l}$ may be approximated by an integral ([9], Chapter III, §1, Corollary 1). More precisely, we have

$$
\Gamma_{l}=\frac{1}{k} \int_{u_{v} / m}^{v / m}(\log y) y^{1 / 4+i t / 2} d y+O\left(\left(\frac{x}{m}\right)^{1 / 4} \mathcal{L}\right) .
$$

Since the character $\chi$ is primitive we have $\sum_{1 \leq l \leq k} \chi(l)=0$. Hence

$$
W_{m} \ll K\left(\frac{x}{m}\right)^{1 / 4} \mathcal{L}, \quad F_{1} \ll D K x^{1 / 4} \mathcal{L} .
$$

The last estimate and (21) imply

$$
E_{10}^{(1)} \ll D K^{3} x^{1 / 4} Y \mathcal{L} .
$$

Using the bounds for $Y$ and $K$ imposed in (19) and (24) and also (2), (25), (27) we get

$$
E_{10}^{(1)} \ll K x^{11 / 8} .
$$

We estimate $E_{10}^{(2)}$ analogously and we obtain

$$
E_{10}^{(2)} \ll K x^{11 / 8} \text {. }
$$

Consider now $E_{10}^{(4)}$. We have

$$
\begin{aligned}
& E_{10}^{(4)} \ll \sum_{k \leq K} \sum_{\chi \bmod k}^{*} \max _{V / 4 \leq v \leq V} \int_{Y / 2}^{Y}\left|\sum_{\substack{m n \leq v \\
m, n>D}} \sum_{\substack{0 \\
m}} a(m) \Lambda(n) \chi(m n)(m n)^{1 / 4+i t / 2}\right| \\
& \ll \mathcal{L}^{2} \max _{\substack{D \leq M, N \leq x / D \\
M N \leq x}} E_{11}, \\
& \times\left(1+\left|L_{2}(t, \alpha)\right|\right) d t
\end{aligned}
$$

where

$(31)$

$$
\begin{aligned}
E_{11} & =E_{11}(V, Y, \alpha, K, M, N) \\
& =\sum_{k \leq K} \sum_{\chi \bmod k}^{*} \max _{V / 4 \leq v \leq V} \int_{Y / 2}^{Y}\left|F^{*}(t)\right|\left(1+\left|L_{2}(t, \alpha)\right|\right) d t,
\end{aligned}
$$




$$
F^{*}(t)=\sum_{\substack{M<m \leq 2 M \\ N<n \leq 2 N \\ m n \leq v}} a(m) \Lambda(n) \chi(m n)(m n)^{1 / 4+i t / 2} .
$$

We may suppose that the maximum in (31) is taken over $v$ of the form $1 / 2+l$ where $l$ is an integer. Applying again Perron's formula we obtain

$$
\begin{aligned}
F^{*}(t)= & \sum_{M<m \leq 2 M} \sum_{N<n \leq 2 N} a(m) \Lambda(n) \chi(m n)(m n)^{1 / 4+i t / 2} \\
& \times\left(\frac{1}{2 \pi i} \int_{\mathcal{L}^{-1}-i x}^{\mathcal{L}^{-1}+i x}\left(\frac{v}{m n}\right)^{s} \frac{d s}{s}+O\left(x^{-1}\left|\log \frac{v}{m n}\right|^{-1}\right)\right) .
\end{aligned}
$$

Hence

$$
F^{*}(t) \ll \mathcal{L} \int_{-x}^{x}|\mathcal{M}| \cdot|\mathcal{N}| \frac{d \tau}{1+|\tau|}+x^{1 / 3}
$$

where

$$
\begin{aligned}
\mathcal{M} & =\sum_{M<m \leq 2 M} a(m) \chi(m) m^{1 / 4-\mathcal{L}^{-1}+i(t / 2-\tau)}, \\
\mathcal{N} & =\sum_{N<n \leq 2 N} \Lambda(n) \chi(n) n^{1 / 4-\mathcal{L}^{-1}+i(t / 2-\tau)} .
\end{aligned}
$$

Formulas (21), (31) and (32) imply

$$
E_{11} \ll \mathcal{L} \int_{-x}^{x} E_{12} \frac{d \tau}{1+|\tau|}+K x^{13 / 12}
$$

where

$$
E_{12}=E_{12}(Y, \alpha, K, M, N, \tau)=\sum_{k \leq K} \sum_{\chi \bmod }^{*} \int_{k / 2}^{Y}|\mathcal{M}| \cdot|\mathcal{N}|\left(1+\left|L_{2}(t, \alpha)\right|\right) d t .
$$

Suppose, for example, that $M \leq N$. Then $M, N$ satisfy

$$
D \leq M \leq x^{1 / 2}, \quad D \leq N \leq x / D, \quad M N \leq x .
$$

By the Cauchy inequality we have

$$
E_{12} \ll\left(E_{13}\right)^{1 / 2}\left(E_{14}\right)^{1 / 2},
$$

where

$$
\begin{aligned}
& E_{13}=E_{13}(Y, \alpha, K, N, \tau)=\sum_{k \leq K} \sum_{\chi \bmod k}^{*} \int_{Y / 2}^{Y}|\mathcal{N}|^{2} d t, \\
& E_{14}=E_{14}(Y, \alpha, K, M, \tau)=\sum_{k \leq K} \sum_{\chi \bmod k}^{*} \int_{Y / 2}^{Y}|\mathcal{M}|^{2}\left(1+\left|L_{2}(t, \alpha)\right|^{2}\right) d t .
\end{aligned}
$$


We estimate $E_{13}$ by Theorem 7.1 of [12] to get

$$
E_{13} \ll \mathcal{L}\left(K^{2} Y+N\right) N^{3 / 2} .
$$

To estimate the integral in the expression for $E_{14}$ we use Theorem 6.1 of [12] and also Theorem 1 of [3]. We obtain

$$
\int_{Y / 2}^{Y}|\mathcal{M}|^{2}\left(1+\left|L_{2}(t, \alpha)\right|^{2}\right) d t \ll x^{\varepsilon} M^{3 / 2} Y,
$$

hence

$$
E_{14} \ll x^{\varepsilon} M^{3 / 2} K^{2} Y
$$

We use (2), (24), (25), (30), (33)-(37) to get

$$
E_{10}^{(4)} \ll K x^{3 / 2-\nu}
$$

for some $\nu>0$. Let us point out that only in this place do we need the tight restriction $\theta<1 / 4-\lambda$.

We proceed with $E_{10}^{(3)}$ analogously to obtain

$$
E_{10}^{(3)} \ll K x^{3 / 2-\nu}
$$

for some $\nu>0$.

We use (24), (26), (28), (29), (38), (39) to find that $E_{9} \ll x^{3 / 2-\nu}$ for some $\nu>0$. The estimation of $E_{8}$ is similar, so we have proved (23) and the proof of the theorem is complete.

Finally, the author would like to thank the referee for his useful remarks.

\section{References}

[1] A. Balog, On the fractional parts of $p^{\theta}$, Arch. Math. (Basel) 40 (1983), 434-440.

[2] - On the distribution of $p^{\theta}(\bmod 1)$, Acta Math. Hungar. 45 (1985), 179-199.

[3] A. Balog and G. Harman, On mean values of Dirichlet polynomials, Arch. Math. (Basel) 57 (1991), 581-587.

[4] E. Bombieri, On the large sieve, Mathematika 12 (1965), 201-225.

[5] E. Bombieri and H. Iwaniec, On the order of $\zeta\left(\frac{1}{2}+i t\right)$, Ann. Scuola Norm. Sup. Pisa 13 (1986), 449-472.

[6] H. Daven port, Multiplicative Number Theory (revised by H. Montgomery), Springer, 1980 .

[7] S. A. Gritsenko, On a problem of I. M. Vinogradov, Mat. Zametki 39 (1986), 625-640 (in Russian).

[8] G. Harman, On the distribution of $\sqrt{p}$ modulo one, Mathematika 30 (1983), 104116.

[9] A. A. Karatsuba and S. M. Voronin, The Riemann Zeta-Function, de Gruyter, 1992.

[10] R. M. Kaufman, The distribution of $\sqrt{p}$, Mat. Zametki 26 (1979), 497-504 (in Russian). 
[11] Yu. V. Linnik, On a theorem in prime number theory, Dokl. Akad. Nauk SSSR 47 (1945), 7-8 (in Russian).

[12] H. L. Montgomery, Topics in Multiplicative Number Theory, Lecture Notes in Math. 227, Springer, 1971.

[13] J. Rivat, Doctoral thesis, Université de Paris-Sud, 1992.

[14] J. Schoissengeier, The connection between the zeros of the $\zeta$-function and sequences $(g(p))$, p prime mod1, Monatsh. Math. 87 (1979), 21-52.

[15] - Eine neue Diskrepanz für gewisse Primzahlfolgen, Sitzungsber. Math.-Naturwiss. Kl., Abt. II, 187 (1978), 219-224.

[16] R. C. Vaughan, An elementary method in prime number theory, Acta Arith. 37 (1980), 111-115.

[17] I. M. Vinogradov, Special Variants of the Method of Trigonometric Sums, Nauka, Moscow, 1976 (in Russian).

Department of Mathematics

Plovdiv University "P. Hilendarski"

Plovdiv 4000, Bulgaria

E-mail: dtolev@ulcc.uni-plovdiv.bg

Received on 30.8.1996

and in revised form on 20.1.1997 